\title{
Eficácia de diferentes inseticidas e de volumes de calda no controle de Spodoptera frugiperda nas culturas do milho e sorgo cultivados em várzea
}

\author{
Efficacy of different insecticides and suspension volumes to control Spodoptera frugiperda \\ in corn and sorghum in low land areas
}

\author{
Marcus Antônio Gonçalves Costa ${ }^{1}$ Anderson Dionei Grützmacher ${ }^{2}$ José Francisco da Silva Martins ${ }^{3}$ \\ Ervandil Correa Costa ${ }^{4}$ Gustavo Storch $^{5}$ Getúlio Jorge Stefanello Júnior ${ }^{6}$
}

\section{RESUMO}

A lagarta-do-cartucho Spodoptera frugiperda (J. E. Smith, 1797) tem causado prejuízos significativos à produtividade nas culturas do milho e sorgo, principalmente em áreas de várzeas. Com o objetivo de determinar-se a eficiência de controle de $\mathbf{S}$. frugiperda em milho e sorgo no agroecossistema de várzea e avaliar a resposta das culturas sobre o rendimento de grãos, foi realizado um experimento na Embrapa Clima Temperado de Pelotas, Estação Experimental de Terras Baixas, Capão do Leão, RS no ano agrícola 2001/2002. Semeou-se milho da cultivar Pioneer $30 \mathrm{~F} 33$ e sorgo da cultivar Aventis BRS $305 \mathrm{em}$ blocos ao acaso com parcelas sub-subdivididas. Os inseticidas utilizados e respectivas dosagens ( $m L$ p.c. ha $^{-1}$ ) foram o Match CE (lufenurom), 300; Rimom 100 CE (novalurom), 150; Tracer SC (espinosade), 50; Lorsbam 480 BR (clorpirifós), 500 e Karate 50 Zeon (lambdacialotrina), 150. As avaliações foram aos 5, 10, 15, 20, e 25 dias após a aplicação. Os volumes de calda $\left(\mathrm{L} \mathrm{ha}^{-1}\right)$ utilizados para cada tratamento foram 0, 150, 200, 250 e 300. A eficiência de controle de $\boldsymbol{S}$. frugiperda com os inseticidas lufenurom, novalurom, espinosade, clorpirifós e lambdacialotrina aplicados em volumes de calda de 0, 150, 200, 250 e 300L ha he $^{-1}$ queriou em função do estádio da cultura do milho e sorgo e época de aplicação. $O$ rendimento de grãos foi independente do volume de calda aplicado tanto para as culturas de milho e sorgo.

Palavras-chave: controle químico, lagarta-do-cartucho.

\begin{abstract}
The fall armyworm Spodoptera frugiperda (J. E. Smith, 1797) has caused significant damage, mainly, in corn and sorghum growing in low land areas. A trial was carried out at Embrapa Clima Temperado de Pelotas, Estação Experimental de Terras Baixas, Capão do Leão, $R S$. The objective was to study the efficacy of $\boldsymbol{S}$. frugiperda control based upon yield response of the corn $\mathrm{cv}$ Pioneer $30 F 33$ and sorghum cv Aventis BRS 305. The experimental design was a random block with split plot. The insecticides and rate were Match CE (lufenurom), $300 \mathrm{~mL}$ p.c. ha $\mathrm{h}^{-1}$; Rimom 100 CE (novalurom), $150 \mathrm{~mL}$ p.c. ha ${ }^{-1}$; Tracer SC (spinosad), $50 \mathrm{~mL}$ p.c. ha ${ }^{-1}$; Lorsbam 480 BR (chlorpyrifos), $500 \mathrm{~mL}$ p.c. ha-1 and Karate 50 Zeon (lambda-cyhalothrin), $150 \mathrm{~mL}$ p.c. ha ${ }^{-1}$. Evaluations were done at 5, 10, 15, 20, and 25 days after application. The volumes of 0, 150, 200, 250 e $300 \mathrm{~L} \mathrm{ha}^{-1}$ were sprayed, respectively, for each treatment. The best control of $\boldsymbol{S}$. frugiperda was obtained with lufenurom, novalurom, spinosad, chlorpyrifos and lambda-cyhalothrin sprayed with 150, 200, 250 e 300L ha${ }^{1}$, respectively, implying that treatment efficacy was independent of volume application. However, treatment efficacy was dependent oN corn and sorghum growth stage and timing of spray. Yield variation was not related to volume application for both, corn and sorghum.
\end{abstract}

Key words: fall armyworm, chemical control.

${ }^{1}$ Programa de Pós-graduação em Fitossanidade, Faculdade de Agronomia "Eliseu Maciel”(FAEM), Universidade Federal de Pelotas (UFPel), Pelotas, RS, Brasil. E-mail: marcusgc@ufpel.tche.br Autor para correspondência.

2Departamento de Fitossanidade, FAEM, UFPel, Campus Universitário, CP 354, 96.010-900, Pelotas, RS, Brasil.

${ }^{3}$ Embrapa Clima Temperado, CP 403, 96.001-970, Pelotas, RS, Brasil.

${ }^{4}$ Departamento de Defesa Fitossanitária, Universidade Federal de Santa Maria (UFSM), Centro de Ciências Rurais (CCR), Campus Universitário, Prédio 42, 97.105-900, Santa Maria, RS.

${ }^{5}$ Programa de Pós-graduação em Fitossanidade, FAEM, UFPel, Pelotas, RS, Brasil.

${ }^{6}$ Curso de graduação em Agronomia, FAEM, UFPel, Pelotas, RS, Brasil. 


\section{INTRODUÇÃO}

A lagarta-do-cartucho Spodoptera frugiperda (Lepidoptera: Noctuidae), é uma das pragas mais importantes da cultura do milho (Zea mays L.) e sorgo (Sorghum vulgare L.), no Brasil (CRUZ \& TURPIN, 1982; ÁVILA et al., 1997; SILVA, 1999) e em outros países (PITRE, 1988; PORTILLO et al., 1994; PORTILLO et al., 1997; CHING'OMA \& PITRE, 1999). Na América Central, principalmente em Honduras PITRE (1986), PORTILLO et al. (1997) e CHING'OMA \& PITRE (1999) descreveram que esse noctuídeo é responsável por prejuízos significativos ao rendimento de grãos tanto nas culturas do milho e sorgo.

No Brasil, os estudos referentes ao controle deste inseto-praga na cultura do sorgo em agroecossistema de várzea, são recentes. Entretanto, existe a preocupação quanto à eficácia do controle químico para evitar casos de resistência da praga a inseticidas. GRÜTZMACHER et al. (2000) evidenciaram que o uso continuado de um mesmo inseticida em um mesmo local é fator preponderante para que ocorra a resistência de $\boldsymbol{S}$. frugiperda, particularmente aos inseticidas piretróides sintéticos, evidenciando que a lambdacialotrina aplicada em milho cultivado em várzea não alcançou um controle eficiente.

No ano agrícola de 1964/65, ALMEIDA et al. (1964) relataram que ocorreu um dos maiores surtos da lagarta-do-cartucho nas culturas do milho, arroz e pastagens sendo que os métodos de controle empregados pelos agricultores, naquela oportunidade, não foram eficientes no combate da praga. SILVA (1999) mencionou alguns casos de ineficiência de inseticidas aplicados na cultura do milho na região de Cruz Alta (RS), especialmente quando a semeadura ocorreu de novembro a janeiro (época tardia), coincidindo com períodos de estiagem.

Vários são os fatores de insucesso no controle de $\boldsymbol{S}$. frugiperda através da aplicação de inseticidas. Neste contexto, o objetivo do trabalho foi estudar a eficácia de alguns inseticidas aplicados em diferentes volumes de calda, nas culturas do milho e sorgo, no agroecossistema de várzea visando o controle da lagarta-do-cartucho.

\section{MATERIAL E MÉTODOS}

Um experimento em blocos ao acaso, com parcelas sub-subdividas e quatro repetições, onde parcelas corresponderam às culturas e subparcelas a inseticidas e volumes de calda, foi instalado na Embrapa Clima Temperado, Capão do Leão, RS, no ano agrícola 2001/2002. As unidades experimentais constituíram-se de quatro fileiras de plantas com $5 \mathrm{~m}$ de comprimento, espaçadas $0,70 \mathrm{~m}$, perfazendo uma área total de $14 \mathrm{~m}^{2}$.

Semeou-se, no dia 20 de dezembro o milho Pioneer 30F33 (ciclo normal) e o sorgo Aventis BRS 305 (granífero com tanino), com densidade de 60 e 310 mil sementes ha $^{-1}$, respectivamente, no sistema de plantio convencional. A emergência das plântulas ocorreu dez dias após a semeadura.

Os tratamentos e as dosagens em $\mathrm{mL}$ p.c. ha ${ }^{-1}$ foram: 1 - lufenurom (Match CE), 300; 2 novalurom (Rimom $100 \mathrm{CE}$ ), 150; 3 - espinosade (Tracer SC), 50; 4 - clorpirifós (Lorsbam 480 BR), 500; 5 lambdacialotrina (Karate 50 Zeon), 150 e 6 - testemunha (somente água). As avaliações foram realizadas aos 5, 10, 15, 20, e 25 dias após a aplicação (DAA), em plantas que apresentavam danos de $\boldsymbol{S}$. frugiperda nas folhas, ou seja, folhas raspadas ou destruídas que formavam os cartuchos, onde posteriormente foi calculada eficiência dos inseticidas através da fórmula de ABBOTT (1925).

Para obtenção dos volumes de calda de 150, 200, 250 e 300 $\mathrm{L} \mathrm{ha}^{-1}$ foram utilizados os bicos Mícron 110XP1,5, Teejet XR 110015, Teejet XR 11002, Teejet XR 11002 sob pressão de 23, 42, 37 e 56 PSI, respectivamente. Foram feitas duas aplicações dos produtos fitossanitários, sendo a primeira realizada aos 19 dias após a emergência (DAE), quando o milho e o sorgo se encontravam no estádio vegetativo $V_{4}$ e $E_{1}$ (quatro folhas completamente expandidas), respectivamente e a segunda aos $47 \mathrm{DAE}$, com as culturas no estádio reprodutivo $V_{10}$ e $E_{4}$ (10 folhas completamente expandidas), respectivamente (VANDERLIP, 2003; RITCHIE et al., 2003).

Em cada unidade amostral formadas por duas linhas centrais de cada subparcela, foi efetuada a colheita de ambas as culturas. As espigas e panículas foram armazenadas em sacos etiquetados determinando-se o rendimento em $\mathrm{kg} \mathrm{ha}^{-1}$ e a umidade corrigida para $13 \%$. Para o rendimento foi realizada a análise de variância e havendo significância entre médias, aplicou-se o teste de Tukey a 5\% de probabilidade de erro, com o auxílio dos programas WinStat e PlotlT 3.2.

\section{RESULTADOS E DISCUSSÃO}

Não ocorreu eficiência mínima de controle (= 80\%) de $\boldsymbol{S}$. frugiperda na cultura do milho por ocasião da primeira aplicação de quaisquer inseticidas e volumes (Tabelas 1 e 2). Na cultura do sorgo, após a primeira aplicação, lufenurom 5 dias após a aplicação com volume de 300L ha ${ }^{-1}$ de calda alcançou eficiência 
Tabela 1 - Porcentagem de plantas danificadas e eficiência de controle da primeira aplicação de inseticidas para o controle de Spodoptera frugiperda, na cultura do milho em terras baixas. Capão do Leão, RS, ano agrícola 2002.

\begin{tabular}{|c|c|c|c|c|c|c|c|c|c|c|c|}
\hline \multirow{3}{*}{ Tratamentos } & \multirow{3}{*}{$\begin{array}{l}\text { Volume } \\
\text { Calda } \\
\left(\mathrm{L} \mathrm{ha}^{-1}\right)\end{array}$} & \multicolumn{10}{|c|}{ Dias após a aplicação } \\
\hline & & \multicolumn{2}{|c|}{5} & \multicolumn{2}{|c|}{10} & \multicolumn{2}{|c|}{15} & \multicolumn{2}{|c|}{20} & \multicolumn{2}{|c|}{25} \\
\hline & & $\mathrm{PD}$ & $\mathrm{C}$ & PD & $\mathrm{C}$ & PD & $\mathrm{C}$ & $\mathrm{PD}$ & $\mathrm{C}$ & $\mathrm{PD}$ & $\mathrm{C}$ \\
\hline \multirow{4}{*}{ Lufenurom } & 150 & 27 & 31 & 28 & 21 & 45 & 22 & 40 & 0 & 43 & 6 \\
\hline & 200 & 13 & 65 & 33 & 0 & 57 & 0 & 41 & 0 & 52 & 0 \\
\hline & 250 & 8 & 77 & 23 & 0 & 45 & 0 & 31 & 24 & 52 & 0 \\
\hline & 300 & 21 & 49 & 23 & 21 & 46 & 25 & 37 & 7 & 60 & 0 \\
\hline \multirow{4}{*}{ Novalurom } & 150 & 26 & 33 & 27 & 21 & 56 & 4 & 52 & 0 & 39 & 15 \\
\hline & 200 & 15 & 59 & 25 & 0 & 54 & 0 & 25 & 26 & 28 & 46 \\
\hline & 250 & 15 & 57 & 10 & 29 & 40 & 0 & 9 & 78 & 19 & 58 \\
\hline & 300 & 28 & 31 & 20 & 31 & 34 & 45 & 38 & 5 & 45 & 0 \\
\hline \multirow{4}{*}{ Espinosade } & 150 & 17 & 56 & 37 & 0 & 51 & 12 & 52 & 0 & 52 & 0 \\
\hline & 200 & 12 & 67 & 30 & 0 & 39 & 0 & 42 & 0 & 45 & 13 \\
\hline & 250 & 9 & 74 & 21 & 0 & 31 & 11 & 29 & 29 & 28 & 38 \\
\hline & 300 & 27 & 34 & 31 & 0 & 47 & 24 & 48 & 0 & 62 & 0 \\
\hline \multirow{4}{*}{ Clorpirifós } & 150 & 16 & 59 & 37 & 0 & 37 & 36 & 51 & 0 & 48 & 0 \\
\hline & 200 & 19 & 49 & 30 & 0 & 30 & 14 & 30 & 12 & 54 & 0 \\
\hline & 250 & 22 & 37 & 41 & 0 & 41 & 0 & 47 & 0 & 55 & 0 \\
\hline & 300 & 35 & 14 & 23 & 21 & 28 & 54 & 51 & 0 & 58 & 0 \\
\hline \multirow{4}{*}{$\begin{array}{l}\text { Lambda- } \\
\text { cialotrina }\end{array}$} & 150 & 19 & 51 & 37 & 0 & 71 & 0 & 59 & 0 & 63 & 0 \\
\hline & 200 & 21 & 43 & 23 & 0 & 57 & 0 & 36 & 0 & 60 & 0 \\
\hline & 250 & 22 & 37 & 30 & 0 & 60 & 0 & 44 & 0 & 53 & 0 \\
\hline & 300 & 12 & 70 & 32 & 0 & 48 & 23 & 46 & 0 & 47 & 0 \\
\hline \multirow{4}{*}{ Testemunha } & 150 & 39 & - & 34 & - & 58 & - & 36 & - & 46 & - \\
\hline & 200 & 37 & - & 24 & - & 35 & - & 34 & - & 52 & - \\
\hline & 250 & 35 & - & 14 & - & 35 & - & 41 & - & 45 & - \\
\hline & 300 & 41 & - & 29 & - & 62 & - & 40 & - & 37 & - \\
\hline
\end{tabular}

\% Plantas danificadas (PD) e \% Controle (C)

de 89\%; aos 10 dias com 250 e 300L ha-1 atingiu $100 \%$ e aos 20 dias após a aplicação com 150 e 200L ha-1 de volume de calda controlou 88 e $100 \%$ da população de S. frugiperda. O inseticida novalurom apresentou uma eficiência no controle da lagarta-do-cartucho em sorgo aos 5 dias quando aplicado em volume de calda de 150 e $200 \mathrm{~L} \mathrm{ha}^{-1}$ atingindo $87 \%$ e $85 \%$, respectivamente. Aos 10 dias todos os volumes utilizados para o controle de $\boldsymbol{S}$. frugiperda atingiram 100\%, com 20 dias após a aplicação de novalurom com volume de 150L ha $^{-1}$, a eficiência foi de $100 \%$ e aos 25 dias este inseticida aplicado em volume de calda de 300 $\mathrm{L} \mathrm{ha}^{-1}$ alcançou $80 \%$. Espinosade determinou eficiência mínima de controle aos 5 dias, aplicado em volume de calda de 150 e 300L ha-1 . Aos 10 dias obteve eficiência máxima com os volumes de 250 e 300L ha-1. Quando avaliado aos 20 dias após a aplicação, obteve novamente eficiência máxima com o maior volume de calda e, aos 25 dias novamente atingiu eficiência mínima com 300L ha-1 . Clorpirifós apresentou eficiência de controle somente aos 10 dias com volume de aplicação de $150 \mathrm{~L} \mathrm{ha}^{-1}$. O inseticida lambdacialotrina propiciou eficiência mínima de controle aos 5 dias após a aplicação com volume de calda de 200L ha-1 (Tabelas 1 e 2).

No estádio reprodutivo do milho que coincidiu com a segunda aplicação, aos 20 dias após a aplicação os inseticidas, lufenurom, novalurom, espinosade e clorpirifós apresentaram eficiência de controle de 85,$86 ; 100,91,94 ; 90 ; 85 \%$, respectivamente, que variou em função do volume de calda aplicado (Tabelas 3 e 4). Na cultura do sorgo, no 
Tabela 2 - Porcentagem de plantas danificadas e eficiência de controle da primeira aplicação de inseticidas para o controle de Spodoptera frugiperda, na cultura do sorgo em terras baixas. Capão do Leão, RS, ano agrícola 2002.

\begin{tabular}{|c|c|c|c|c|c|c|c|c|c|c|c|}
\hline \multirow{3}{*}{ Tratamentos } & \multirow{3}{*}{$\begin{array}{l}\text { Volume } \\
\text { Calda } \\
\left(\mathrm{L} \mathrm{ha}^{-1}\right)\end{array}$} & \multicolumn{10}{|c|}{ Dias após a aplicação } \\
\hline & & \multicolumn{2}{|c|}{5} & \multicolumn{2}{|c|}{10} & \multicolumn{2}{|c|}{15} & \multicolumn{2}{|c|}{20} & \multicolumn{2}{|c|}{25} \\
\hline & & $\mathrm{PD}$ & $\mathrm{C}$ & $\mathrm{PD}$ & $\mathrm{C}$ & $\mathrm{PD}$ & $\mathrm{C}$ & $\mathrm{PD}$ & $\mathrm{C}$ & $\mathrm{PD}$ & $\mathrm{C}$ \\
\hline \multirow{4}{*}{ Lufenurom } & 150 & 27 & 31 & 28 & 21 & 45 & 22 & 40 & 0 & 43 & 6 \\
\hline & 200 & 13 & 65 & 33 & 0 & 57 & 0 & 41 & 0 & 52 & 0 \\
\hline & 250 & 8 & 77 & 23 & 0 & 45 & 0 & 31 & 24 & 52 & 0 \\
\hline & 300 & 21 & 49 & 23 & 21 & 46 & 25 & 37 & 7 & 60 & 0 \\
\hline \multirow{4}{*}{ Novalurom } & 150 & 26 & 33 & 27 & 21 & 56 & 4 & 52 & 0 & 39 & 15 \\
\hline & 200 & 15 & 59 & 25 & 0 & 54 & 0 & 25 & 26 & 28 & 46 \\
\hline & 250 & 15 & 57 & 10 & 29 & 40 & 0 & 9 & 78 & 19 & 58 \\
\hline & 300 & 28 & 31 & 20 & 31 & 34 & 45 & 38 & 5 & 45 & 0 \\
\hline \multirow{4}{*}{ Espinosade } & 150 & 17 & 56 & 37 & 0 & 51 & 12 & 52 & 0 & 52 & 0 \\
\hline & 200 & 12 & 67 & 30 & 0 & 39 & 0 & 42 & 0 & 45 & 13 \\
\hline & 250 & 9 & 74 & 21 & 0 & 31 & 11 & 29 & 29 & 28 & 38 \\
\hline & 300 & 27 & 34 & 31 & 0 & 47 & 24 & 48 & 0 & 62 & 0 \\
\hline \multirow{4}{*}{ Clorpirifós } & 150 & 16 & 59 & 37 & 0 & 37 & 36 & 51 & 0 & 48 & 0 \\
\hline & 200 & 19 & 49 & 30 & 0 & 30 & 14 & 30 & 12 & 54 & 0 \\
\hline & 250 & 22 & 37 & 41 & 0 & 41 & 0 & 47 & 0 & 55 & 0 \\
\hline & 300 & 35 & 14 & 23 & 21 & 28 & 54 & 51 & 0 & 58 & 0 \\
\hline \multirow{4}{*}{$\begin{array}{l}\text { Lambda- } \\
\text { cialotrina }\end{array}$} & 150 & 19 & 51 & 37 & 0 & 71 & 0 & 59 & 0 & 63 & 0 \\
\hline & 200 & 21 & 43 & 23 & 0 & 57 & 0 & 36 & 0 & 60 & 0 \\
\hline & 250 & 22 & 37 & 30 & 0 & 60 & 0 & 44 & 0 & 53 & 0 \\
\hline & 300 & 12 & 70 & 32 & 0 & 48 & 23 & 46 & 0 & 47 & 0 \\
\hline \multirow{4}{*}{ Testemunha } & 150 & 39 & - & 34 & - & 58 & - & 36 & - & 46 & - \\
\hline & 200 & 37 & - & 24 & - & 35 & - & 34 & - & 52 & - \\
\hline & 250 & 35 & - & 14 & - & 35 & - & 41 & - & 45 & - \\
\hline & 300 & 41 & - & 29 & - & 62 & - & 40 & - & 37 & - \\
\hline
\end{tabular}

\% Plantas danificadas (PD) e \% Controle (C)

estádio reprodutivo $\left(E_{4}\right)$, a infestação de plantas por $\boldsymbol{S}$. frugiperda foi menor na testemunha em relação aos tratamentos, favorecendo a eficiência dos inseticidas aplicados. Assim, aos 5 dias após a aplicação, destacaram-se os inseticidas lufenurom, novalurom e espinosade, aplicados com volumes de 150, 200 e 300, 150 , onde as eficiências atingidas foram de 100, 100 e $80,80 \%$, respectivamente. Ressalta-se que a partir dos 10 dias após a segunda aplicação o sorgo iniciou o processo de emissão de panículas, desfavorecendo assim o habitat da praga que reside principalmente no cartucho (Tabelas 3 e 4).

Ocorreram variações na eficiência de controle, que provavelmente se justificam em função do estádio de desenvolvimento da praga, pressão populacional, época de aplicação dos inseticidas e tecnologia de aplicação (Tabelas de 1 a 4). Assim, ALMEIDA et al. (1964) relataram que a utilização de bicos leque Teejet 6502, permitiram maior eficiência dos inseticidas no controle de $\boldsymbol{S}$. frugiperda, devido ao menor ângulo de abertura do jato. SILVA (1999) obteve melhor resultado no controle de $\boldsymbol{S}$. frugiperda quando utilizou bico leque 8004.

Apesar da queda na população da praga em ambas as culturas aos 5 DAA, a infestação, na cultura do milho, permaneceu, no entanto, acima do nível de dano estabelecido para o estádio vegetativo considerado o qual é de $20 \%$ de plantas com folhas 
Tabela 3 - Porcentagem de plantas danificadas e eficiência de controle da segunda aplicação de inseticidas para o controle de Spodoptera frugiperda, na cultura do milho em terras baixas. Capão do Leão, RS, ano agrícola 2002.

\begin{tabular}{|c|c|c|c|c|c|c|c|c|c|c|c|}
\hline \multirow{3}{*}{ Tratamentos } & \multirow{3}{*}{$\begin{array}{l}\text { Volume } \\
\text { Calda } \\
\left(\mathrm{L} \mathrm{ha}^{-1}\right)\end{array}$} & \multicolumn{10}{|c|}{ Dias após a aplicação } \\
\hline & & \multicolumn{2}{|c|}{5} & \multicolumn{2}{|c|}{10} & \multicolumn{2}{|c|}{15} & \multicolumn{2}{|c|}{20} & \multicolumn{2}{|c|}{25} \\
\hline & & PD & $\mathrm{C}$ & $\mathrm{PD}$ & $\mathrm{C}$ & $\mathrm{PD}$ & $\mathrm{C}$ & $\mathrm{PD}$ & $\mathrm{C}$ & PD & $\mathrm{C}$ \\
\hline \multirow{4}{*}{ Lufenurom } & 150 & 68 & 0 & 36 & 29 & 27 & 56 & 3 & 85 & 5 & 29 \\
\hline & 200 & 54 & 8 & 27 & 49 & 16 & 63 & 1 & 96 & 12 & 33 \\
\hline & 250 & 40 & 29 & 30 & 25 & 17 & 68 & 6 & 68 & 16 & 0 \\
\hline & 300 & 61 & 0 & 37 & 7 & 26 & 57 & 5 & 54 & 5 & 74 \\
\hline \multirow{4}{*}{ Novalurom } & 150 & 61 & 0 & 45 & 12 & 27 & 56 & 0 & 100 & 8 & 0 \\
\hline & 200 & 47 & 20 & 16 & 70 & 23 & 47 & 2 & 91 & 10 & 44 \\
\hline & 250 & 32 & 44 & 16 & 60 & 11 & 79 & 1 & 94 & 8 & 20 \\
\hline & 300 & 60 & 0 & 15 & 62 & 28 & 53 & 7 & 36 & 6 & 68 \\
\hline \multirow{4}{*}{ Espinosade } & 150 & 63 & 0 & 33 & 35 & 27 & 56 & 2 & 90 & 17 & 0 \\
\hline & 200 & 46 & 22 & 43 & 19 & 37 & 15 & 10 & 56 & 10 & 44 \\
\hline & 250 & 63 & 0 & 40 & 0 & 39 & 28 & 7 & 63 & 6 & 40 \\
\hline & 300 & 63 & 0 & 40 & 0 & 47 & 22 & 13 & 0 & 11 & 42 \\
\hline \multirow{4}{*}{ Clorpirifós } & 150 & 56 & 3 & 56 & 0 & 53 & 14 & 3 & 85 & 7 & 0 \\
\hline & 200 & 68 & 0 & 34 & 35 & 36 & 18 & 11 & 52 & 9 & 50 \\
\hline & 250 & 58 & 0 & 39 & 2 & 57 & 0 & 16 & 16 & 14 & 0 \\
\hline & 300 & 63 & 0 & 31 & 22 & 43 & 28 & 5 & 54 & 9 & 52 \\
\hline \multirow{4}{*}{$\begin{array}{l}\text { Lambda- } \\
\text { cialotrina }\end{array}$} & 150 & 59 & 0 & 54 & 0 & 59 & 4 & 16 & 23 & 4 & 43 \\
\hline & 200 & 52 & 11 & 53 & 0 & 41 & 6 & 9 & 61 & 7 & 61 \\
\hline & 250 & 63 & 0 & 41 & 0 & 48 & 11 & 20 & 0 & 13 & 0 \\
\hline & 300 & 49 & 14 & 47 & 0 & 52 & 13 & 25 & 0 & 7 & 63 \\
\hline \multirow{4}{*}{ Testemunha } & 150 & 58 & - & 51 & - & 62 & - & 21 & - & 7 & - \\
\hline & 200 & 59 & - & 53 & - & 44 & - & 23 & - & 18 & - \\
\hline & 250 & 57 & - & 40 & - & 54 & - & 19 & - & 10 & - \\
\hline & 300 & 57 & - & 40 & - & 60 & - & 11 & - & 19 & - \\
\hline
\end{tabular}

\% Plantas danificadas (PD) e \% Controle (C)

raspadas 30 dias após a semeadura (SIFUENTES, 1967). Esse fato, porém, não foi observado na cultura do sorgo, que apresentou decréscimo de plantas atacadas após $5 \mathrm{DAA}$, permanecendo durante todo o desenvolvimento fenológico, sob infestação reduzida de $\boldsymbol{S}$. frugiperda (Figura 1).

Ocorreu decréscimo da população de $\boldsymbol{S}$. frugiperda, após a segunda aplicação de inseticidas, estando relacionado principalmente ao estádio de desenvolvimento do milho do que a eficiência dos inseticidas empregados (Figura 2). O florescimento do milho provavelmente tenha prejudicado a praga, pois o cartucho foi substituído pelo pendão, deslocando a praga do seu local considerado ótimo para o seu desenvolvimento, fazendo com que provavelmente, essa ficasse exposta aos inimigos naturais dos fatores ambientas. Observou-se por ocasião da avaliação do dano em milho que as lagartas de $\boldsymbol{S}$. frugiperda, deslocaram-se para a base das espigas, situando-se, entre o colmo da planta e a base da espiga recémformada. Não raro foram observados ataques na ponta da espiga. As lagartas destruíram nesse caso, o estiloestigma e freqüentemente penetravam para o interior das espigas recém-formadas, concordando com ÁVILA et al. (1997). Aos 25 DAA (segunda aplicação), ocorreu um novo pico populacional de $\boldsymbol{S}$. frugiperda nessa cultura, permanecendo acima do nível de dano preconizado e que, nesse estádio fenológico, é de $10 \%$ 
Tabela 4 - Porcentagem de plantas danificadas e eficiência de controle da segunda aplicação de inseticidas para o controle de Spodoptera frugiperda, na cultura do sorgo em terras baixas. Capão do Leão, RS, ano agrícola 2002.

\begin{tabular}{|c|c|c|c|c|c|c|c|c|c|c|c|}
\hline \multirow{3}{*}{ Tratamentos } & \multirow{3}{*}{$\begin{array}{l}\text { Volume } \\
\text { Calda } \\
\left(\mathrm{L} \mathrm{ha}^{-1}\right)\end{array}$} & \multicolumn{10}{|c|}{ Dias após a aplicação } \\
\hline & & \multicolumn{2}{|c|}{5} & \multicolumn{2}{|c|}{10} & \multicolumn{2}{|c|}{15} & \multicolumn{2}{|c|}{20} & \multicolumn{2}{|c|}{25} \\
\hline & & $\mathrm{PD}$ & $\mathrm{C}$ & $\mathrm{PD}$ & $\mathrm{C}$ & $\mathrm{PD}$ & $\mathrm{C}$ & $\mathrm{PD}$ & $\mathrm{C}$ & PD & $\mathrm{C}$ \\
\hline \multirow{4}{*}{ Lufenurom } & 150 & 0 & 100 & 0 & 100 & 0 & 100 & 0 & 100 & 0 & 100 \\
\hline & 200 & 1 & 66 & 1 & 50 & 0 & 100 & 0 & 100 & 0 & 100 \\
\hline & 250 & 1 & 0 & 0 & 100 & 0 & 100 & 0 & 100 & 0 & 100 \\
\hline & 300 & 4 & 20 & 0 & 100 & 0 & 100 & 0 & 100 & 0 & 100 \\
\hline \multirow{4}{*}{ Novalurom } & 150 & 4 & 20 & 0 & 100 & 0 & 100 & 0 & 100 & 0 & 100 \\
\hline & 200 & 0 & 100 & 0 & 100 & 0 & 100 & 0 & 100 & 0 & 100 \\
\hline & 250 & 2 & 0 & 0 & 100 & 0 & 100 & 0 & 100 & 0 & 100 \\
\hline & 300 & 1 & 80 & 0 & 100 & 0 & 100 & 0 & 100 & 0 & 100 \\
\hline \multirow{4}{*}{ Espinosade } & 150 & 1 & 80 & 0 & 100 & 0 & 100 & 0 & 100 & 0 & 100 \\
\hline & 200 & 3 & 0 & 1 & 0 & 0 & 100 & 0 & 100 & 0 & 100 \\
\hline & 250 & 2 & 0 & 2 & 0 & 0 & 100 & 0 & 100 & 0 & 100 \\
\hline & 300 & 2 & 60 & 1 & 50 & 0 & 100 & 0 & 100 & 0 & 100 \\
\hline \multirow{4}{*}{ Clorpirifós } & 150 & 6 & 0 & 2 & 0 & 1 & 0 & 0 & 100 & 0 & 100 \\
\hline & 200 & 4 & 0 & 2 & 0 & 1 & 0 & 0 & 100 & 0 & 100 \\
\hline & 250 & 3 & 0 & 1 & 0 & 0 & 100 & 0 & 100 & 0 & 100 \\
\hline & 300 & 7 & 0 & 2 & 0 & 1 & 0 & 0 & 100 & 0 & 100 \\
\hline \multirow{4}{*}{$\begin{array}{l}\text { Lambda- } \\
\text { cialotrina }\end{array}$} & 150 & 2 & 60 & 0 & 100 & 0 & 100 & 0 & 100 & 0 & 100 \\
\hline & 200 & 6 & 0 & 3 & 0 & 0 & 100 & 0 & 100 & 0 & 100 \\
\hline & 250 & 2 & 0 & 2 & 0 & 0 & 100 & 0 & 100 & 0 & 100 \\
\hline & 300 & 3 & 40 & 1 & 50 & 0 & 100 & 0 & 100 & 0 & 100 \\
\hline \multirow{4}{*}{ Testemunha } & 150 & 5 & - & 2 & - & 1 & - & 0 & - & 0 & - \\
\hline & 200 & 3 & - & 1 & - & 0 & - & 0 & - & 0 & - \\
\hline & 250 & 0 & - & 0 & - & 0 & - & 0 & - & 0 & - \\
\hline & 300 & 5 & - & 2 & - & 0 & - & 0 & - & 0 & - \\
\hline
\end{tabular}

\% Plantas danificadas (PD) e \% Controle (C)

de folhas raspadas quando as plantas têm de 40 a 60 dias (Figura 2). Esses resultados corroboram com aqueles obtidos por WAQUIL et al. (1982) e BUNTIN (1986), os quais constataram que os ataques de $\boldsymbol{S}$. frugiperda podem ocorrer em quaisquer estádios de desenvolvimento do milho, além de evidenciarem que uma maior densidade populacional desse inseto-praga propiciou uma infestação tardia, mesmo a cultura estando em pleno estádio reprodutivo.

A análise da variância para o rendimento de grãos e a interação entre esse parâmetro na cultura e volume de calda foi significativo ( $\mathrm{P} \leq 0,0085)$. Verificou-se que o rendimento tanto para as duas culturas manteve-se constante, independente do volume de calda aplicado, onde milho e sorgo apresentaram rendimento médio de 625 e 1.215 $\mathrm{kg} \mathrm{ha}^{-1}$, respectivamente. Esses resultados aproximaram-se aos obtidos por SILVA et al. (2002), os quais em um estudo do espaçamento e da população de sorgo granífero cultivar BRS 305, cultivado em solo de várzea subtropical, obtiveram um rendimento de $1.330 \mathrm{~kg} \mathrm{ha}^{-1}$ quando o espaçamento empregado foi de $0,68 \mathrm{~m}$.

De acordo com o teste de Tukey não houve diferença significativa entre o rendimento de grãos da testemunha em relação aos demais tratamentos em ambas as culturas. Quando analisadas as culturas, o teste demonstrou ser significativa a diferença. Deste modo, pode-se inferir que inseticidas e volumes 


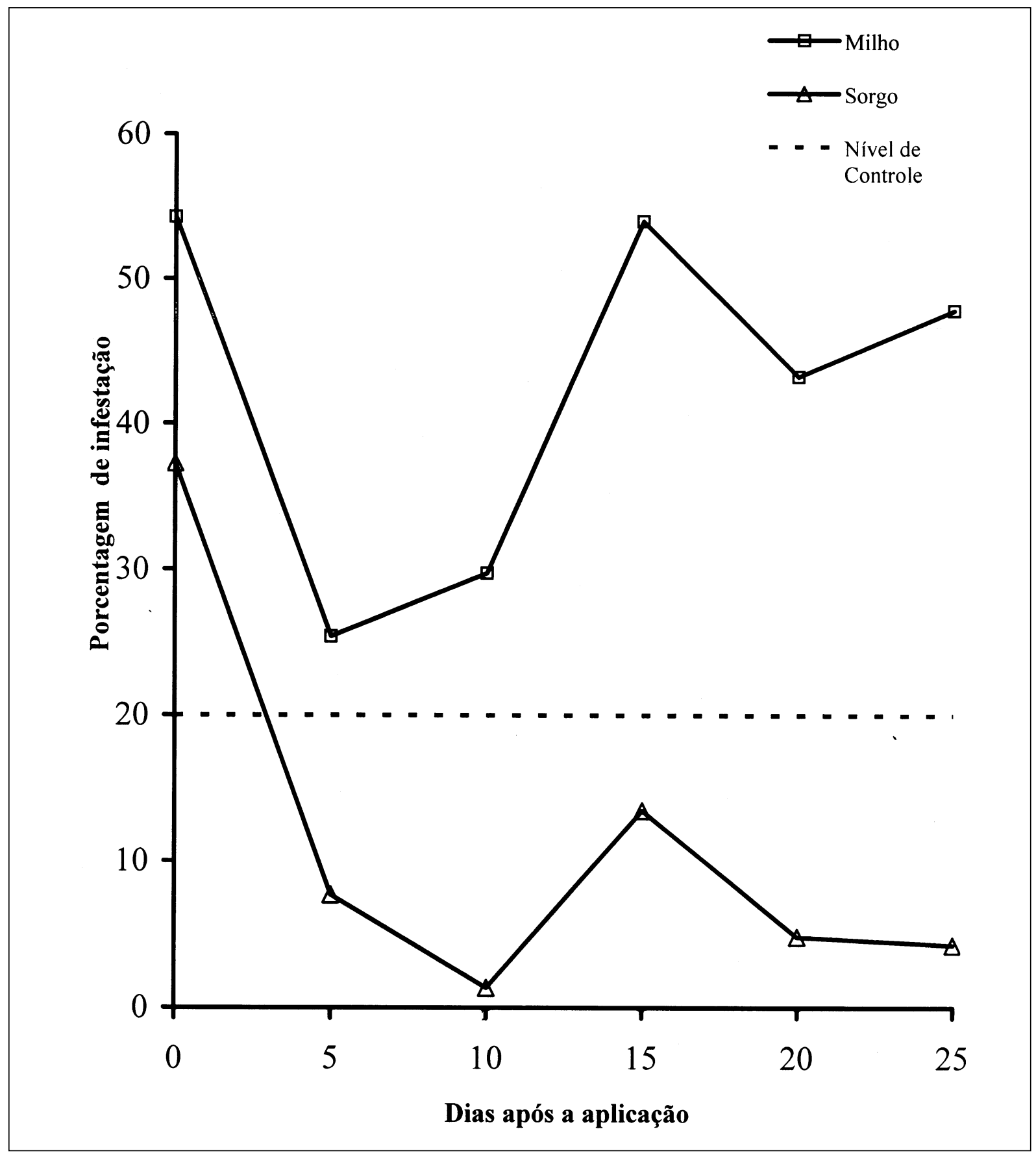

Figura 1- Porcentagem de infestação de Spodoptera frugiperda nas culturas do milho e sorgo após a primeira aplicação. Capão do Leão, RS, ano agrícola 2002.

de calda não influenciaram no controle de $\boldsymbol{S}$. frugiperda.

Sendo também um dos pontos da discussão o volume de calda utilizada no controle de $\boldsymbol{S}$. frugiperda, tendo em vista o aumento da produtividade, os dados obtidos neste trabalho são de fato incontroversos, de forma que o volume de calda utilizado demonstrou claramente que não é o fator essencial quando se trata de controle. Fica afastada, portanto, a hipótese de que volume de calda seja o elemento essencial, sendo apenas um veículo pelo qual o ingrediente ativo atinge o seu objeto-alvo. 


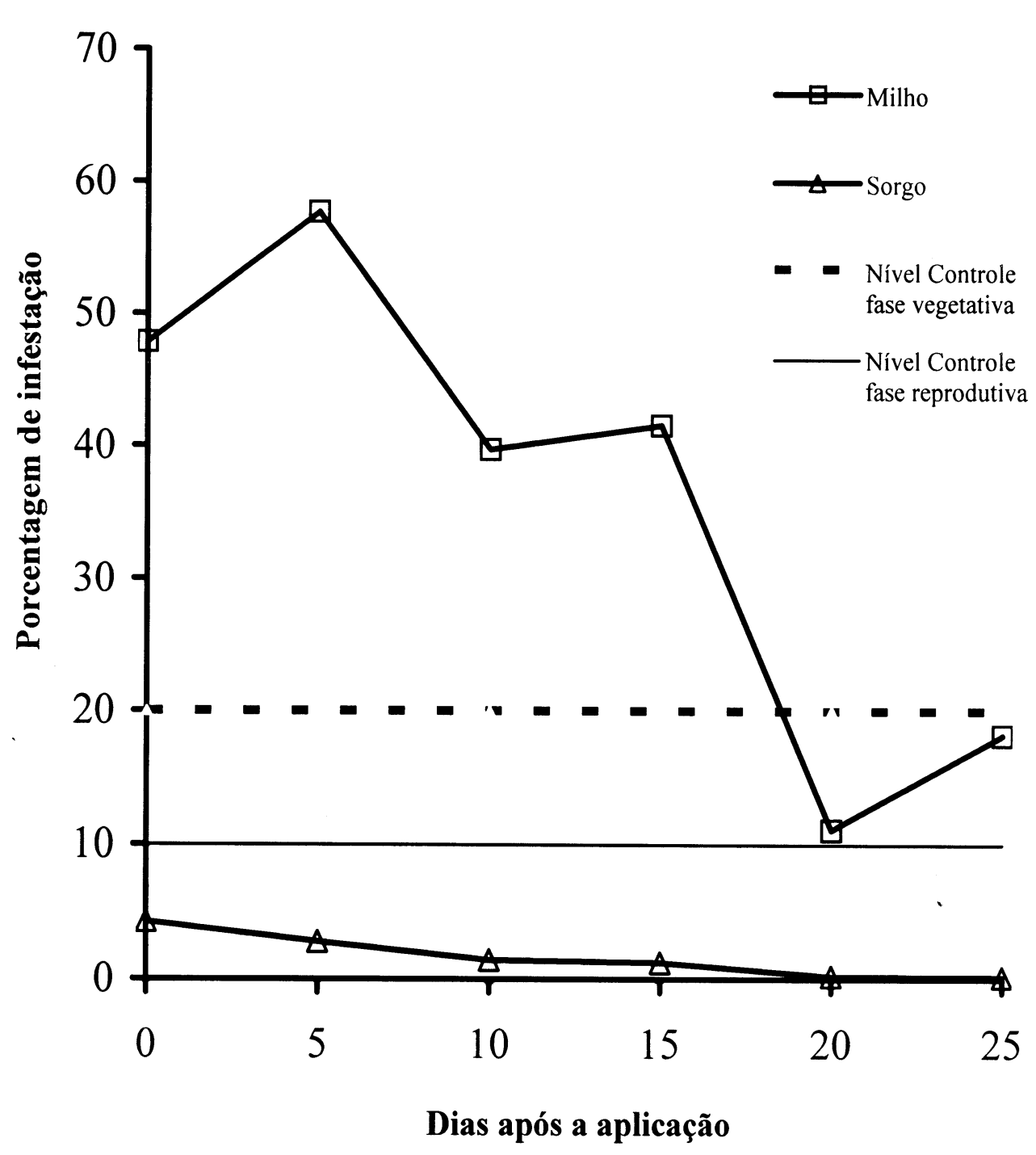

Figura 2 - Porcentagem de infestação de Spodoptera frugiperda nas culturas do milho e sorgo após a segunda aplicação. Capão do Leão, RS, ano agrícola 2002.

\section{CONCLUSÕES}

A eficácia de controle de Spodoptera frugiperda quando aplicados os inseticidas lufenurom, novalurom, espinosade, clorpirifós e lambdacialotrina em volumes de calda de 150, 200, 250 e 300L ha-1 variam em função do estádio fenológico das culturas do milho e sorgo e época de aplicação. O rendimento de grãos é independente do volume de calda aplicado com diferentes inseticidas no controle de $\boldsymbol{S}$. frugiperda para as culturas do milho e do sorgo.

\section{AGRADECIMENTOS}

Apoio: bolsa de estudos do Conselho Nacional de Desenvolvimento Científico e Tecnológico (CNPq). Bolsistas Doutorado CNPq: Costa, M.A.G.; Storch, G. Bolsista Iniciação Científica, CNPq: Stefanello Júnior, G.J. Pesquisadores do CNPq: Grützmacher, A.D.; Costa, E.C.

\section{REFERÊNCIAS}

ABBOTT, W.S. A method of computing the effectiveness of an insecticide. Journal of Economic Entomology, Lanham, v.18, n.1, p.265-267, 1925.

Ciência Rural, v.35, n.6, nov-dez, 2005. 
ALMEIDA, P.R. et al. Ensaio com inseticidas modernos no combate à lagarta-do-cartucho Laphygna frugiperda (Smith \& Abbot, 1797) e técnica de aplicação. O Biológico, São Paulo, v.30, n.5, p.111-114, 1964.

ÁVILA, C.J. et al. Insetos pragas: reconhecimento, comportamento, danos e controle. Dourados: Embrapa-CPAO, 1997. 24p. (Embrapa-CPAO. Circular Técnica, 5).

BUNTIN, G.D. A review of plant response to fall armyworm, Spodoptera frugiperda (J.E. Smith), injury to select field and forage crops. Florida Entomologist, Gainesville, v.69, n.3, p.549-559, 1986.

CHING'OMA, G.P.; PITRE, H.N. Oviposition by fall armyworm, Spodoptera frugiperda (J. E. Smith): effects of moth age and sorghum maturation stage. Ceiba, Honduras v.40, n.1, p.57-62, 1999.

CRUZ, C.; TURPIN, F.T. Efeito da Spodoptera frugiperda em diferentes estádios de crescimento da cultura do milho. Pesquisa Agropecuária Brasileira, Brasilia, v.17, n.3, p.355-359, 1982.

GRÜTZMACHER, A.D. et al. Efeito de inseticidas e de tecnologias de aplicação no controle da lagarta-do-cartucho na cultura do milho no agroecossistema de várzea. In: REUNIÃO TÉCNICA ANUAL DO SORGO, 28., REUNIÃO TÉCNICA ANUAL DO MILHO, 45., 2000, Pelotas, RS. Anais... Pelotas: Embrapa de Clima Temperado, 2000. p.567573. CD ROM.

PITRE, H.N. Chemical control of the fall armyworm (Lepidoptera: Noctuidae): an update. Florida Entomologist, Gainesville, v.69, n.3, p.570-578, 1986.

PITRE, H.N. A complex of lepidopterous defoliators on sorghum and maize in southern Honduras. Ceiba, Honduras, v.29, n.2, p.353-361, 1988.
PORTILLO, H.E. et al. Improved chemical protection of sorghum seed and seedlings form insect pests in Honduras. Turrialba, Turrialba, Costa Rica, v.44, n.1, p.50-55, 1994.

PORTILLO, H.E. et al. Validation of new agronomic and plant protection technologies in intercropped sorghum and maize in southern Honduras. Ceiba, Honduras, v.38, n.2, p.35-43, 1997.

RITCHIE, W.R. et al. How a corn plant develops. Capturado em 06 jul, 2003. Online. Disponível na Internet http://www.ag.iastate.edu/departments/agronomy/ corngrows.html.

SIFUENTES, A.J.A. Oviposición de palomillas de cogollero y daño de las larvas en plántulas de maíz y sorgo, en invernadero. Agricultura Técnica en México, México, Chapingo, v.2, n.7, p.311-314, 1967.

SILVA, M.T.B. Fatores que afetam a eficiência de inseticidas sobre Spodoptera frugiperda Smith em milho. Ciência Rural, Santa Maria, v.29, n.3, p.383-387, 1999.

SILVA, C.A.S. et al. Estudo do espaçamento e da população para sorgo granífero, cultivado em solo de várzea subtropical. In: REUNIÃOO TÉCNICA, DIVERSIFICAÇÃO DO USO DE VÁRZEAS DE CLIMA TEMPERADO, 2002, Pelotas, RS. Anais... Pelotas: Embrapa Clima Temperado, 2002. p.145152. (Documentos, 90).

VANDERLIP, R.L. How a sorghum plant develops. Kansas State University, January 1993. Capturado em 30 ago, 2003. On line. Disponível na Internet http://www.oznet.ksu.edu/ library/crpsl2/s3.pdf.

WAQUIL, J.M. et al. Controle da lagarta-do-cartucho em milho com inseticidas químicos e biológicos. Pesquisa Agropecuária Brasileira, Brasília, v.17, n.2, p.163-166, 1982. 